\title{
Relación entre el período de lactancia materna y maloclusiones
}

\section{Resumen}

Los beneficios de la lactancia materna en la prevención de las maloclusiones han sido descritos en la literatura odontológica, destacando que lactar exclusivamente más de 6 meses disminuye su prevalencia. Objetivo: determinar la relación entre periodo de Lactancia Materna y Maloclusiones. Material y métodos: se examinó una muestra de 59 niños en edades entre 6 y 13 años en la parroquia Higuerote, Miranda, Venezuela. Se aplicó un cuestionario a cada representante para obtener datos sobre el periodo de lactancia materna recibida por el niño y se efectuó un examen clínico bucal observando variables ortodóncicas en cada niño. Se realizó un modelo de análisis de significancia $\mathrm{p}<0,05$, mediante la prueba de Fisher a través del Análisis de Varianza Univariado (ANOVA), para evidenciar si existían diferencias estadísticamente sig-
Rondón, Rosa Gabriela ${ }^{1}$

Zambrano, Gabriel Alejandro ${ }^{2}$

Guerra, María Elena ${ }^{3}$ nificativas entre periodo de lactancia materna y las variables: relación molar permanente, mordida abierta anterior y mordida cruzada posterior. También se aplicó la prueba no paramétrica de Kruskal-Wallis en aquellos casos en donde la muestra fue menor de 30 observaciones. Resultados: existe relación estadística significativa entre un periodo de lactancia materna menor de 6 meses y relación molar en clase II de Angle y mordida abierta anterior, sin embargo para mordida cruzada posterior no se halló significancia estadística. Se encontró relación estadísticamente significativa entre un periodo de lactancia materna mayor de 6 meses con relación molar en clase I de Angle. Conclusión: se plantea que un periodo de lactancia materna mayor de 6 meses se relaciona con la ausencia de maloclusión en el grupo estudiado.

Palabras clave: lactancia materna, maloclusión.

\section{Artigo Original}

\section{Relação entre 0 aleitamento materno e má oclusão}

\section{Resumo}

Os benefícios da amamentação na prevenção da má oclusão têm sido descritos na literatura dental, notando que amamentam exclusivamente seis meses sobre a sua prevalência diminui. $\mathbf{O b}-$ jetivo: determinar a relação entre o aleitamento materno e má oclusão. Métodos: Uma amostra de 59 crianças com idade entre 6 e 13 anos na paróquia Higuerote, Miranda, Venezuela. Um

\footnotetext{
${ }^{1}$ Odontóloga, Universidad Central de Venezuela. Práctica Privada.

${ }^{2}$ Odontólogo, Universidad Central de Venezuela. Residente de la Especialización en Odontología Infantil. Universidad Central de Venezuela.

${ }^{3}$ Doctora en Ciencias, mención Salud Bucal Materno Infantil. Profesora Titular, Universidad Central de Venezuela.
} 
questionário foi aplicado para cada representante para obter dados sobre o período de amamentação fornecidas pela criança e fez um exame oral em variáveis ortodônticos observando cada criança. Realizamos um modelo de análise de significância $\mathrm{p}<0,05$, pelo teste de Fisher por meio de análise de variância univariada (ANOVA), para mostrar se houve diferenças estatisticamente significativas entre a amamentação e variáveis maternas: razão molar permanente, mordida aberta anterior e mordida cruzada posterior. Também aplicado o teste não paramétrico de Kruskal-Wallis nos casos em que a amostra foi de menos de 30 observações. Resultados:
Não há relação significativa entre o aleitamento materno até 6 meses e razão molar e Angle classe II mordida aberta anterior, mordida cruzada posterior no entanto, para não haver significância estatística foi encontrada. Relação estatisticamente significativa foi encontrada entre o período de aleitamento materno superior a 6 meses na classe I razão molar de Angle. Conclusão: sugere-se que o período de amamentação durante 6 meses está relacionada com a ausência de má oclusão no grupo de estudo.

Palavras-chave: aleitamento materno, má oclusão.

Original article

\section{Relations between breastfeeding and malocclusion}

\begin{abstract}
The benefits of breastfeeding in the prevention of malocclusion have been described in dental literature, noting that exclusively breastfeeding over 6 months reduced its prevalence. Objetive: To determine the relationship between breastfeeding and malocclusions. Materials and methods: We examined a sample of 59 children aged between 6 and 13 years in the parish Higuerote, Miranda, Venezuela. A questionnaire was applied to each tutor to obtain data on the breastfeeding period received by the child and was made an oral examination and orthodontic variables was observed in each child. We performed an analysis model of significance $p<0.05$, by Fisher test through the Univariate Analysis of Variance to reveal whether there were signifi-
\end{abstract}

cant differences between maternal breastfeeding and the variables: permanent molar relationship, anterior open bite and posterior crossbite. Also applied the nonparametric Kruskal-Wallis test in cases where the sample was less than 30 observations. Results: A statistically significant relationship exists between a breastfeeding less than 6 months and Angle Class II molar relation and anterior open bite, but for posterior crossbite was not found statistically significant. Statistically significant relationship was found between maternal breastfeeding over 6 months with Angle Class I molar relation. Conclusion: it is suggested that a period of breastfeeding over 6 months is related to the absence of malocclusion in the study group.

Keywords: Breastfeeding, malocclusion. 


\section{Introducción}

La Organización Mundial de la Salud señala que la lactancia materna es una práctica natural y constituye la forma idónea de aportar a los niños pequeños todos los nutrientes necesarios para un crecimiento y desarrollo adecuado y saludable ${ }^{1}$. Posee efectos indiscutibles para la salud de la madre como del hijo desde el punto de vista inmunológico ${ }^{2,3}$, nutritivo ${ }^{1}$, afectivo, y psicológico $^{4,5}$. Se recomienda que la alimentación materna debe ser exclusiva los primeros 6 meses de vida y luego debe ser complementada con otros alimentos por lo menos hasta los 2 años. ${ }^{1}$

Aun cuando la promoción de la lactancia materna se ha hecho a gran escala, la misma no se inicia tan pronto como debería, la exclusividad y la frecuencia no se están logrando por los periodos recomendados, la duración sigue siendo corta (menor a 6 meses) y las prácticas de destete siguen siento inadecuadas. ${ }^{6}$

Todas las funciones del aparato bucal (respiración, succión, deglución, masticación y fonación), estimulan el crecimiento y desarrollo del complejo dento-buco-máxilo-facial. Por ello, el tipo de lactancia, así como los hábitos parafuncionales, pueden influir sobre la morfología definitiva de los maxilares, ocasionando disturbios en la oclusión. ${ }^{7}$

La importancia de la lactancia materna en el adecuado crecimiento y desarrollo cráneo-facial, así como su relación con la apropiada maduración de las funciones del aparato bucal, han sido descritas en la literatura odontológica. Es significativo destacar el aporte de Raymonds, J. (2003), quien acota que la lactancia materna posee un papel primordial en el desarrollo adecuado del lactante, específicamente en el crecimiento, desarrollo y maduración del macizo facial y en consecuencia su insuficiencia se relaciona como uno de los agentes etiológicos de determinadas maloclusiones, ${ }^{8}$ por lo que un tiempo mayor de 6 meses de lactancia materna contribuye a prevenir las maloclusiones, o al menos disminuir su gravedad.

Numerosos trabajos de investigación Latinoamericanos relacionan un periodo de lactancia materna corto (menor de 6 meses) con tendencia a relación molar en clase II de Angle, $, 10,12,13,14,15$ mordida abierta anterior ${ }^{9,12,14,16,17,18}$ y mordida cruzada posterior. ${ }^{16,19,20,21}$

Lo anterior nos motivó a estudiar si existe correspondencia entre el periodo de lactancia materna y la relación molar en clase II de Angle, la mordida abierta anterior y la mordida cruzada posterior en una muestra de niños en estado de pobreza crítica que habitan en la parroquia $\mathrm{Hi}$ guerote, municipio Brion del estado Miranda, Venezuela.

\section{Material y métodos}

La población de este estudio estuvo constituida por 59 niños en periodo de dentición mixta en edades comprendidas entre 6 y 13 años que habitan el sector "La Isla de la Fantasía" en la parroquia Higuerote, municipio Brion, estado Miranda, Venezuela, quienes decidieron voluntariamente en conjunto con sus representantes ser parte de la investigación con el fin de estimar el porcentaje de niños amamantados por 6 meses o menos que presentan relación molar en clase II de Angle, mordida abierta anterior y / o mordida cruzada posterior. La evaluación clínica y medición odontológica de los niños fueron realizadas por odontólogos egresados de la Universidad Central de Venezuela previo entrenamiento.

Para la recolección de datos, se aplicó un instrumento adaptado de la historia clínica de la 
Cátedra de Odontología Pediátrica de la UCV, que constaba de dos partes: un interrogatorio y un examen clínico,bajo entrenamiento previo, el cual fue evaluado y aceptado por la tutora. A todos los niños se les realizó un minucioso examen odontológico con el fin de obtener componentes clínicos relacionados con el desarrollo adecuado de los maxilares y a sus representantes se les suministró una encuesta con el fin de determinar variables sociodemográficas y periodo de lactancia materna recibido por el menor. Para la recopilación de la información fueron reunidos los representantes de los niños y se les explicó el objetivo de la investigación, aquellos niños cuyos tutores legales accedieron voluntariamente a participar fueron incluidos en el estudio, bajo consentimiento informado firmado con testigos.

El tipo de estudio realizado es de tipo clínico, experimental no probabilístico, de corte transversal. y de carácter correlacional.

El análisis estadístico de las variables se realizó mediane un modelo de análisis de significancia $\mathrm{p}<0,05$, basados en la prueba de Fisher a través del Análisis de Varianza Univariado (ANOVA), para evidenciar si existían diferencias estadísticamente significativas entre periodo de lactancia materna recibida por el infante y las variables: relación molar permanente, mordida abierta anterior y mordida cruzada posterior. Y prueba no paramétrica de Kruskal-Wallis en aquellos casos donde la muestra fue menor de 30 observacio-

\section{DISTRIBUCIÓN DE LA POBLACIÓN POR PERÍODO DE LACTANCIA MATERNA}

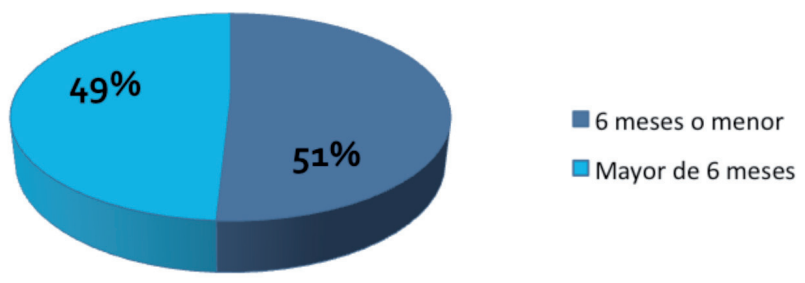

Gráfico 1. Distribución de la población estudiada por periodo de lactancia materna recibida.

nes, igualmente con el mismo nivel de significancia $\mathrm{p}<0,05$.

\section{Resultados}

El 100\% de los niños participantes en este estudio recibieron lactancia materna por algún tiempo. De los cuales el $51 \%$ fue amamantado por un periodo de 6 meses o menor, observándose una mediana de tiempo de lactancia de 6 meses (Ver Gráfico 1). Las relaciones molares en la población estudiada se observan en la Tabla 1.

Para la relación molar en clase I de Angle se encontró relevancia estadística en la población lactada por más de 6 meses a diferencia a las relaciones molares en clase II y clase III de Angle.

En cuanto a la relación molar en Clase II de Angle sólo se observó en 17 niños (28,8\%), de los cuales, el $71 \%$ fue lactado por un periodo me-

Tabla 1. Relación molar permanente en la población estudiada acorde con la duración de la lactancia materna.

\begin{tabular}{|l|c|c|c|}
\hline & Lactancia materna & Lactancia materna & Total \\
\hline Relación Molar Clase I de Angle & $15(25.4 \%)$ & $20(33.9 \%)$ & $35(59.3 \%)$ \\
\hline Relación Molar Clase II de Angle & $12(20.3 \%)$ & $5(8.5 \%)$ & $17(28.8 \%)$ \\
\hline Relación Molar Clase III de Angle & $3(2.4 \%)$ & $4(8.5 \%)$ & $7(11.9 \%)$ \\
\hline
\end{tabular}

Fuente: Propia. 
Tabla 2. Relación del periodo de lactancia materna con las variables: mordida abierta anterior y mordida cruzada posterior.

\begin{tabular}{|c|c|c|c|}
\hline & $\begin{array}{c}\text { Lactancia materna } \\
\mathbf{6} \text { meses }\end{array}$ & $\begin{array}{c}\text { Lactancia materna } \\
>6 \text { meses }\end{array}$ & Total \\
\hline Mordida Abierta Anterior & $7(11.9 \%)$ & $5(8.5 \%)$ & $12(20.4 \%)$ \\
\hline Mordida Cruzada posterior & $2(3.4 \%)$ & $1(1.7 \%)$ & $3(5.1 \%)$ \\
\hline $\begin{array}{l}\text { Mordida Abierta Anterior y } \\
\text { Mordida Cruzada Posterior }\end{array}$ & $5(8.5 \%)$ & $0(0 \%)$ & $5(8.5 \%)$ \\
\hline
\end{tabular}

Fuente: Propia.

nor de 6 meses, resultado que arrojó relevancia significativa cuando se le aplicó la prueba no paramétrica de Kruskal-Wallis a través del Análisis de Varianza Univariado (ANOVA) con un nivel del significancia $\mathrm{p}<0,05 \mathrm{y}$ de confiabilidad del $95 \%$. Hallazgo que indica que un periodo de lactancia materna menor de 6 meses se relaciona con tendencia a distoclusión.

En relación a las otras variables estudiadas: mordida abierta anterior, mordida cruzada posterior y la combinación de mordida abierta anterior y mordida cruzada posterior, se encontró mayor prevalencia de estas en aquellos niños que fueron lactados por un periodo menor de 6 meses, sin embargo al aplicar la prueba no paramétrica de Kruskal-Wallis sólo se encontró diferencia estadística significativa en la variable mordida abierta anterior, cuando se le relacionó con el grupo lactado por más de 6 meses y con el total de esta categoría. $\mathrm{p}<0,05$ Estos resultados pueden ser apreciados en la Tabla 2.

\section{Discusión}

En la literatura odontológica se ha descrito que la etiología de las maloclusiones es multifactorial en la cual se combinan la genética y factores ambientales. Autores consideran que la lac- tancia materna ejerce una gran influencia en el adecuado crecimiento y desarrollo del aparato bucal. $6,7,8,19,22,23,24,25$ De igual manera varios autores concuerdan en que la misma genera un efecto positivo en la sincronización de las funciones del aparato bucal: succión, deglución, respiración, masticación y fonación. 6,8,30,31,32

Algunos de los autores consultados afirman que la alimentación por medio de la lactancia materna desde el nacimiento y por un tiempo mayor a 6 meses contribuye notablemente a la prevención de alteraciones dento-buco-máxilofaciales. $78,12,16,19,25,26,27,28,29,30$ Por el contrario, los infantes que son alimentados por medio del biberón, desde el nacimiento o son destetados antes de los 6 meses de vida presentan 13 veces más posibilidades de sufrir maloclusiones sobretodo asociadas a hábitos viciosos de succión y de deglución. ${ }^{14}$

Al igual que en este estudio, Mendoza, A. \& Cols. (2008), Blanco, L. \& Cols. (2007), Prieto, Y. \& Cols. (2009), Guerra, M. \& Cols. (2010) y Pérez, M. \& Cols. (2008), concluyen en sus investigaciones que un periodo de lactancia materna menor a 6 meses constituye un factor predisponente en la aparición de maloclusiones. . $^{4,28,29,35,39}$

De acuerdo a lo revisado en la literatura, las maloclusiones más frecuentes encontradas en pa- 
cientes que han recibido lactancia materna por un periodo menor de seis meses son: la relación molar en clase II o distoclusión, 7,10,12,13,15 mordida abierta anterior ${ }^{12,14,18,21,33}$ y mordida cruzada posterior. ${ }^{17,19,20,21,33,34}$ Sin embargo, Cristiane, L \& Cols. (2006) en un estudio realizado sobre 249 niños brasileros no hallaron una relación estadística significativa entre la duración de la lactancia materna exclusiva y la prevalencia de distoclusión. ${ }^{11}$ De igual manera, Legovic, M. \& Col (1991) en su investigación realizada en niños croatas concluyeron que no existían diferencias relevantes entre la prevalencia de Maloclusión Clase II de Angle en niños amamantados y no amamantados. ${ }^{35}$

Los resultados de nuestro trabajo arrojaron que si existía una relación estadística significativa entre un periodo de lactancia materna corto (menor de 6 meses) y la relación molar en clase II de Angle y la mordida abierta anterior, lo que concuerda con la mayoría de las investigaciones realizadas a nivel mundial. En la muestra tomada también se consideró la mordida cruzada posterior, pero con la misma no se halló relevancia estadística. De igual forma se encontró una relación estadísticamente significativa entre un periodo de lactancia materna mayor de 6 meses con relación molar en clase I de Angle.
En relación a la maloclusión Clase III de Angle no se encontró relevancia estadística en ninguno de los grupos estudiados.

\section{Conclusiones}

Se encontró una relación estadística significativa entre un periodo de lactancia materna exclusiva corto (de 6 meses o menor) y una relación molar permanente en Clase II de Angle y en menor proporción con mordida abierta anterior.

No se halló relevancia de mordida cruzada posterior en la población estudiada con respecto a un periodo de lactancia de 6 meses o menor.

En los resultados obtenidos también se pudo apreciar que un periodo de lactancia materna mayor de 6 meses se asocia con relación molar permanente Clase I de Angle mostrando una prevalencia muy alta si se le compara con Clase II y III de Angle.

\section{Recomendación}

Continuar realizando estudios en el área en los que se tomen en consideración también hábitos viciosos de succión y de deglución en relación con su prevalencia en niños amamantados por un periodo de 6 meses o menor.

\section{Referencias}

1. WHO (World Health Organization): (2001) The optimal duration of exclusive breast feeding: results of a WHO systematic review. Indian Pediatric. 38: 565-7.

2. Lawrence, RA; Lawrence, RM: (2010) Breastfeeding: a guide for the medical profession. 7ma Edición, Filadelfia.

3. Ip S, Chung M, Raman G, Chew P, Magula N, DeVine D, Trikalinos T, Lau J: (2007) Breastfeeding and Maternal and Infant Health Outcomes in Developed Countries. Evidence Report/Technology Assessment No. 153 (Prepared by Tufts-New England Medical Center Evidence-based Practice Center, under Contract No. 290-02-0022). AHRQ Publication No. 07-E007. Rockville, MD:Agency for Healthcare Research and Quality.

4. Chien PF, Howie PW: (2001) Breast milk and the risk of opportunistic infection in infancy in industrialized and nonindustrialized settings. Adv Nutr Res 2001(10):69-104.

5. Bai YK, Middlestadt SE, Joanne Peng CY, Fly AD: (2009) Psychosocial factors underlying the mother's decision to continue exclusive breastfeeding for 6 months: an elicitation study. J Hum Nutr Diet 2009(22):134-140. 
6. Cujiño, M: (2004) Lactancia materna: factor protectivo de la dentición. Hacia la promoción de la salud. Vol. 9:45-51. Obtenible en: http: / / promocionsalud.ucaldas.edu.co/downloads / Revista\%209_6.pdf

7. Mendoza, A; Asbún, P; Crespo, A; González, S; Patiño, R: (2008) Relación de la lactancia materna y hábitos de succión no nutritiva con maloclusión dental. Rev. bol. ped. Vol.47(1) La Paz Ene. 2008.

8. Raymonds, J: (2003) La lactancia y la buena mordida. Revista Colombiana de Pediatría. Obtenible en: http:/ / www. encolombia.com/medicina/pediatria/ pedi39104-liga.htm

9. Ortega, G: (1998) Ventajas de la lactancia materna para la salud bucodental. Rev Cubana Ortod.Vol.13(1):53-54.

10. Blanco, L; Guerra, ME; Mujica, C: (1999) Relación entre el amamantamiento: el tipo del perfil: oclusión y hábitos viciosos en preescolares. Arch. venez. pueric. pediatr. Vol.62(3):138-143.

11. Cristiane, L; Garib, D; Arouca, R: (2006) Association between breastfeeding duration and mandibular retrusion: A cross-sectional study of children in the mixed dentition. American Journal of Orthodontics and Dentofacial Orthopedics. Vol. 130(4): 531-534.

12. Blanco, L; Guerra, ME; Rodríguez, S: (2007) Lactancia materna y maloclusiones dentales en preescolares de la Gran Caracas. Acta Odontológica Venezolana. Vol. 45(2) Caracas 2007.

13. Prieto, Y; Alfaro, E; Malavé, C: (2009) Influencia del Periodo de Lactancia en la Presencia de Maloclusiones. Universidad Nacional Experimental "Rómulo Gallegos”, San Juan de los Morros, Venezuela. Obtenible en: http: / / iadr.confex. com/iadr/venez09/preliminaryprogram/abstract_126901.htm

14. Guerra, ME; Rodríguez, S; Blanco, L: (2010) Relationship Between Breastfeeding Period and Dentobuccomaxillofacial Characteristics in Venezuelan Children. IADR. July 14-17,2010. Obtenible en: http: / / iadr.confex.com/iadr / 2010barce/ preliminaryprogram/abstract_131547.htm

15. Thomas, E; Cangussu, M, Assis, A: (2012) Maternal breastfeeding, parafunctional oral habits and malocclusion in adolescents: A multivariate analysis. Int J Pediatr Otorhinolaryngol. 2012 Feb 4.

16. Lopez, L; Singh, D; Feliciano, N; Machuca, M: (2006) Associations between a history of breastfeeding, malocclusion and parafunctional habits in Puerto Rican children. PRHSJ. Vol. 25(1):31-34.

17. Glazer, K; Barros, A; Peres, M; Victora, C: (2007) Effects of breastfeeding and sucking habits on malocclusion in a birth cohort study. Rev. Saúde Pública. Vol.41(3) São Paulo June 2007.

18. Oliveira, A; Pordeus, I; Torres, C; Martins, M; Paiva, S: (2010) Feeding and non-nutritive sucking habits and prevalence of open bite and crossbite in children/adolescents with Down Syndrome. Angle Orthod. Vol.80(4):748-753.

19. Ferrer, A; Villalba, V: (2006) Effect of the suction-swallowing action on orofacial development and growth. Rev Fac Cien Med Univ Nac Cordoba. Vol.63(2):33-37.

20. Kobayashi, H; Scavone, H; Ferreira, R; Garib, D: (2010) Relationship between breastfeeding duration and prevalence of posterior crossbite in the deciduous dentition. Am J Orthod Dentofacial Orthop. Vol.137(1):54-58.

21. Ferras, I; Pereira, E; Pereira, A; De Castro, M; Nóbilo, K; Bovi, G: (2010) Breastfeeding, deleterious oral habits and malocclusion in 5-year-old children in São Pedro, SP, Brazil. Dental Press J. Orthod. Vol. 15( 2):71-81.

22. Medeiros, A; Ferreira, J; Felicio, C: (2009) Correlation between feeding methods, non- nutritive sucking and orofacial behaviors. Pro Fono. Vol.21(4):315-319.

23. Campaña, V: (2012) La lactancia y su relación con la presencia de maloclusiones en niños de 0 a 6 años con discapacidad. Universidad Nacional de Cuyo, Mendoza, Argentina. Obtenible en: http:/ /www.portalesmedicos.com/publicaciones / articles / 3934 / 1/ La-lactancia-y-su-relacion-con-la-presencia-de-maloclusiones-en-ninos-de-0-a-6-anos-condiscapacidad.html

24. Merino, E: (2003) Lactancia materna y su relación con las anomalías dentofaciales. Acta Odontológica Venezolana. Vol. 41(2): 42-46.

25. Benitez, M; Calvo, L; Quirós, O; Maza, P; D Jurisic, A; Alcedo, C; Fuenmayor, D: (2009) Estudio de la lactancia materna como un factor determinante para prevenir las anomalías dentomaxilofaciales. Revista Latinoamericana de Ortodoncia y Odontopediatria “Ortodoncia.ws edición electrónica septiembre 2009. Obtenible en: www.ortodoncia.ws. Consultada: 20/02/2012. 
26. López, Y; Arias, M; Zelenenko, O: (1999) Lactancia materna en la prevención de anomalías dentomaxilofaciales. Rev. Cubana Ortod. Vol. 14(1): 32-38.

27. Guerra, ME; Mujica, C: (1999) Influencia del amamantamiento en el desarrollo de los maxilares. Acta Odontológica Venezolana. Vol. 37(2)/1999.

28. Romero, C; Scavone, H; Gamba, D; Cotrim, F; Ferreira, R: (2011) Breastfeeding and non-nutritive sucking patterns related to the prevalence of anterior open bite in primary dentition. J. Appl. Oral Sci. Vol.19(2). Bauru Mar./ Apr. 2011

29. Vasconcelos, F; De Lima, A; Vilela, M; Brito, A; Tornisiello, C; Rosenblatt: (2011) Non-nutritive sucking habits, anterior open bite and associated factors in Brazilian children aged 30-59 months. Braz. Dent. J. Vol.22(2). Ribeirão Preto 2011.

30. Campaña, V: (2012) La lactancia y su relación con la presencia de maloclusiones en niños de 0 a 6 años con discapacidad. Universidad Nacional de Cuyo, Mendoza, Argentina. Obtenible en: http:/ /www.portalesmedicos.com/publicaciones / articles / 3934 / 1/ La-lactancia-y-su-relacion-con-la-presencia-de-maloclusiones-en-ninos-de-0-a-6-anos-condiscapacidad.html

31. Guerra, ME; Blanco, L; Mujica, C: (2006) Relación entre período de amamantamiento y desarrollo maxilar en niños indígenas pemones venezolanos. Bol. Asoc. Argent. Odontol. Niños. Vol.35(1):11-14.

32. Perez, M; Quiroga, M: (2008) Prevención de maloclusiones a partir de la promoción de la lactancia materna y la educación para el control de hábitos. Pontificia Universidad Javeriana de Bogotá. Obtenible en: http:// recursostic. javeriana.edu.co/wiki/index.php/Prevenci\%C3\%B3n_de_Maloclusiones_a_partir_de_la_promoci\%C3\%B3n_de_la_ lactancia_materna_y_la_educaci\%C3\%B3n_para_el_control_de_h\%C3\%A1bitos

33. Medina, C; Laboren, M; Viloria, C; Quirós, O; D’Jurisic, A; Alcedo, C; Molero, L; Tedaldi, J: (2010) Hábitos bucales más frecuentes y su relación con Malocusiones en niños con dentición primaria. Revista Latinoamericana de Ortodoncia y Odontopediatria “Ortodoncia.ws” edición electrónica julio 2010. Obtenible en: www.ortodoncia.ws. Consultada, $20 / 02 / 2012$.

34. Oliveira, A; Paiva, S; Torres, M; Silva, C; Almeida, I: (2011) Prevalence and determinant factors of malocclusion in children with special needs. Eur J Orthod. Vol.33(4):413-418.

35. Legovic, M; Ostric, L: (1991) The effects of feeding methods on the growth of jaws in infants. J. Dent. Child. N ${ }^{\circ} 58$ : 253-255.

Recibido: 26-07-12

Aceptado: 12-09-12

Correspondencia: marielena05@gmail.com 